\title{
On the distance scale of open clusters
}

\author{
A.V. Loktin ${ }^{\star}$ \\ Astronomical observatory of the Ural Federal University, 51 Lenin Street, Yekaterinburg 620000, Russia
}

Received 2013 Apr 3, accepted 2013 May 28

Published online 2013 Oct 1

Key words open clusters and associations: general - techniques: photometric

We discuss the state of the distance scale of open clusters in view of various capabilities provided by optical and nearIR observations of galactic objects. Various checks lead to the conclusion that open cluster distance scale requires no appreciable correction.

\section{Introduction}

Open clusters $(\mathrm{OCl})$ constitute one of the most important classes of objects used to investigate the structure and evolution of the Milky Way because they allow simultaneous estimation of the heliocentric distance and age. Since the 1980s we have been developing computer programs for estimating the main cluster parameters from photometric data with the aim to construct catalogues of cluster parameters that should be homogeneous in terms of the methods employed, the distance and age scales. The application of the first such program resulted in the creation of the first version of the "Homogeneous Catalogue of Open Cluster Parameters" (Loktin \& Matkin 1990), and the second set of programs brought about the next version of the catalogue (Loktin, Gerasimenko \& Malisheva 2001), which we use until now. However, time has come to develop a new set of programs to make use of near-infrared data. Large-scale IR-surveys (2MASS, UKIDSS, VISTA) are playing ever increasing part in galactic research. These surveys provide the researchers with huge amounts of homogeneous photometry, which is used, in particular, in studies of the parameters of open clusters.

\section{Usage of 2MASS data}

The first our attempt of extensive determination of $\mathrm{OCl}$ parameters based on the 2MASS Point Source Catalogue (Skrutskie et al. 2006) data started two years ago. It involved estimating the color excesses, distance moduli, and ages of clusters believed to belong to the Perseus spiral arm. The aim of this study was to enrich the cluster sample in this region and then use it to investigate galactic spiral structure. The main parameters have been determined for a total of 240 clusters. We determined cluster reddening using the $Q_{J H K}-(J-H)$ diagram, which we found to be the best

\footnotetext{
^ Corresponding author: Alexhander.Loktin@usu.ru
}

choice among available photometrical diagrams. We compare in Figs. 1 and 2 the new estimates of cluster parameters to those of the current version of Homogeneous catalogue. As is evident from the figures, the IR reddening and age estimates do not deviate appreciably from the corresponding optical estimates, whereas there is an evident discrepancy between the optical and near-IR scales of distance moduli (see Fig. 2). Note that this discrepancy between the distance scales cannot be attributed to reddening, but should be due only to the isochrone fitting procedure.

The relation between the two sets of distance modulus estimates appears to be nonlinear. In Fig. 2 we approximate this relation by two straight lines, which show the two distance scales to be nearly coincident at moderate distances, and the clusters located beyond $1000 \mathrm{pc}$ to lie far from the $45^{\circ}$ bisector. Such a behavior of the distance scales led us to verify the cluster distance scale using all available methods. This effect can be caused by many factors, but we first had to check the correctness of the distance scale of the "Homogeneous catalogue".

Figure 3 presents one of the methods of verifying the cluster distance scale. It shows 161 young clusters (log $t<$ 7.5 , dots) from the "Homogeneous Catalogue" plotted in the coordinates $(\ln R, \theta)$ where logarithmic spirals become straight lines. Here $R$ is the galactocentric distance, and $\theta$ the galactocentric angle. The solid line shows the position of the Orion spiral arm inferred using the least squares method with clusters we suppose to belong to this arm. The open circles show the positions of maser sources with distances determined from the published trigonometric parallaxes based on VLBA radio observations.

It is evident from the plot that the spiral arms outlined by open clusters coincide with the arms outlined by the maser sources with no appreciable shift (see, in particular, the Perseus complex with $\ln R \simeq 2.28, \theta=0.2 \mathrm{rad}$ ), where heliocentric distances of the clusters significantly exceed $1 \mathrm{kpc}$. 

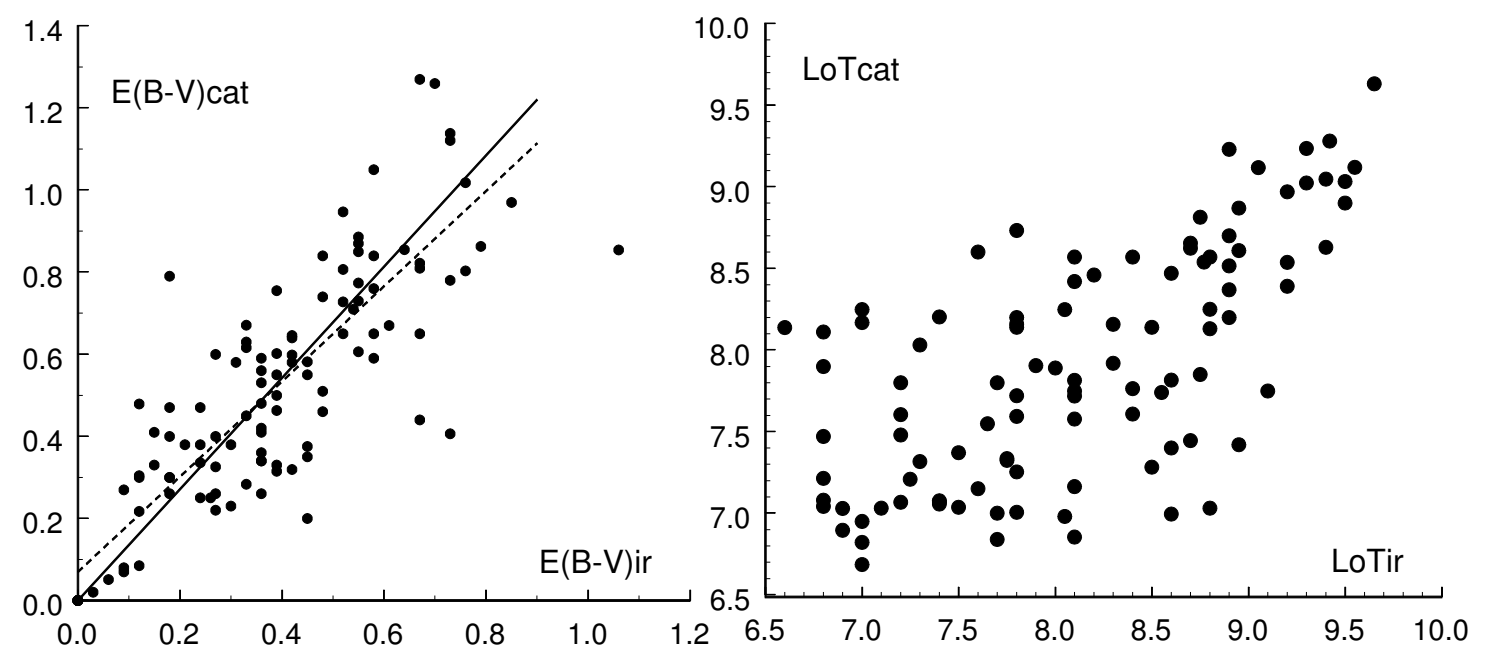

Fig. 1 Comparison between the color excess (left panel) and logarithms of age (right panel) estimates for open clusters based on optical (the "cat" subscript) and near IR (the "ir" subscript) photometry. The lines in the left panel are the $45^{\circ}$ bisector (the solid line) and the least squares solution (dashed line).

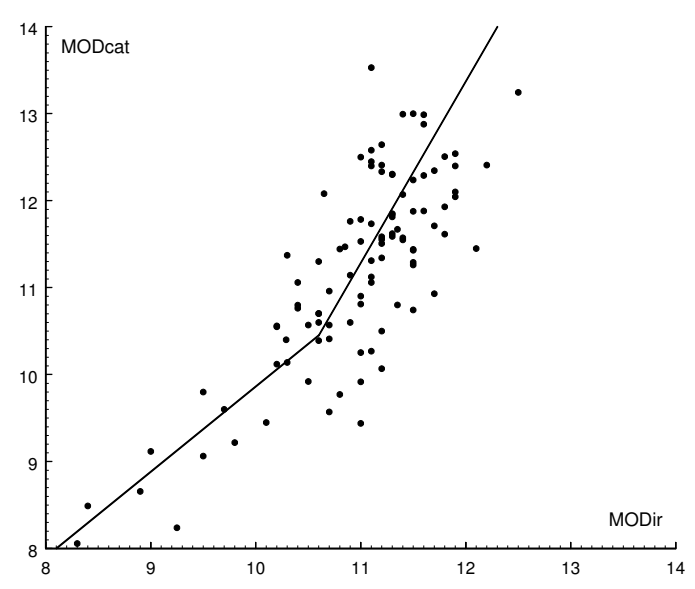

Fig. 2 Comparison of the estimates of distance moduli for optical and IR sets of data.

The revised Hipparcos trigonometric parallaxes (van Leeuwen 2007) also provide a check for the cluster distance scale. We calculated the trigonometric parallax based distances for as many clusters as possible. To this end, we selected all clusters with previous distance determinations $r<1000 \mathrm{pc}$, which provided us finally with the distance estimates for a total of 68 clusters. For each cluster we selected its probable members based on Hipparcos proper motions, CMD and $Q-(J-H)$ photometric diagrams. Figure 4 compares the new parallax-based distances with those from the "Homogeneous Catalogue".

The solid line in the right panel of Fig. 4 is the $45^{\circ}$ bisector. The plots in Fig. 4 show the distance scale of the "Homogeneous Catalogue" clusters to be consistent with Hipparcos trigonometric parallaxes.

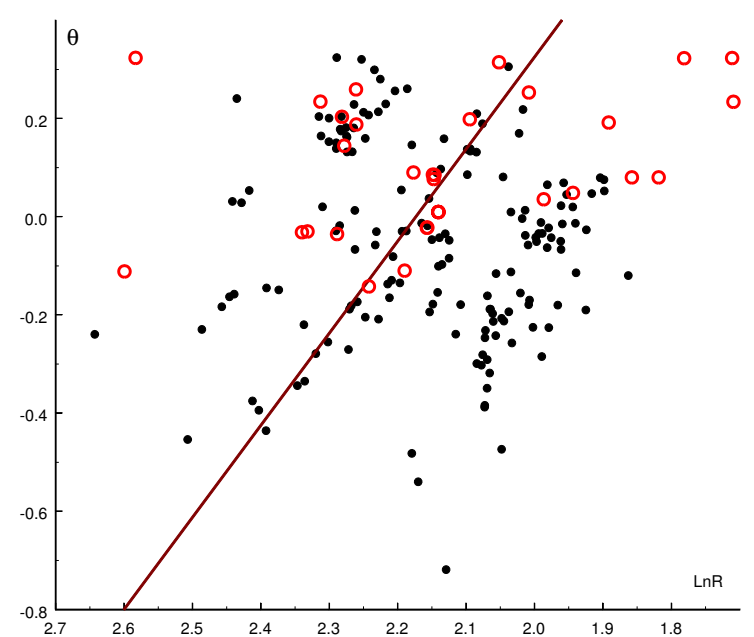

Fig. 3 The agreement of spiral structure fragments as identified from the positions of open clusters (filled circles) and maser sources (open circles) with VLBI parallax based distances.

Yet another check for the distance scale is provided by the approach used by Twarog, Anthony-Twarog \& Edgington-Giordano (2009). These authors approximated a short portion of ZAMS using Hipparcos trigonometric parallaxes for stars with available metallicity determinations. They showed the position of ZAMS on optical colourmagnitude diagrams to shift substantially with metallicity in the interval of metallicities appropriate for open clusters. It is interesting, among other things, to see whether the corresponding shift is significant for near-IR photometry.

We used for our ZAMS fit the metallicities from the PASTEL catalogue (Soubiran et al. 2010) supplemented with 2MASS JHKs magnitudes and Hipparcos trigonometrical parallaxes. We rejected stars with $J$ and $K$ photometric errors greater than 0.1 and stars with trigonometric paral- 


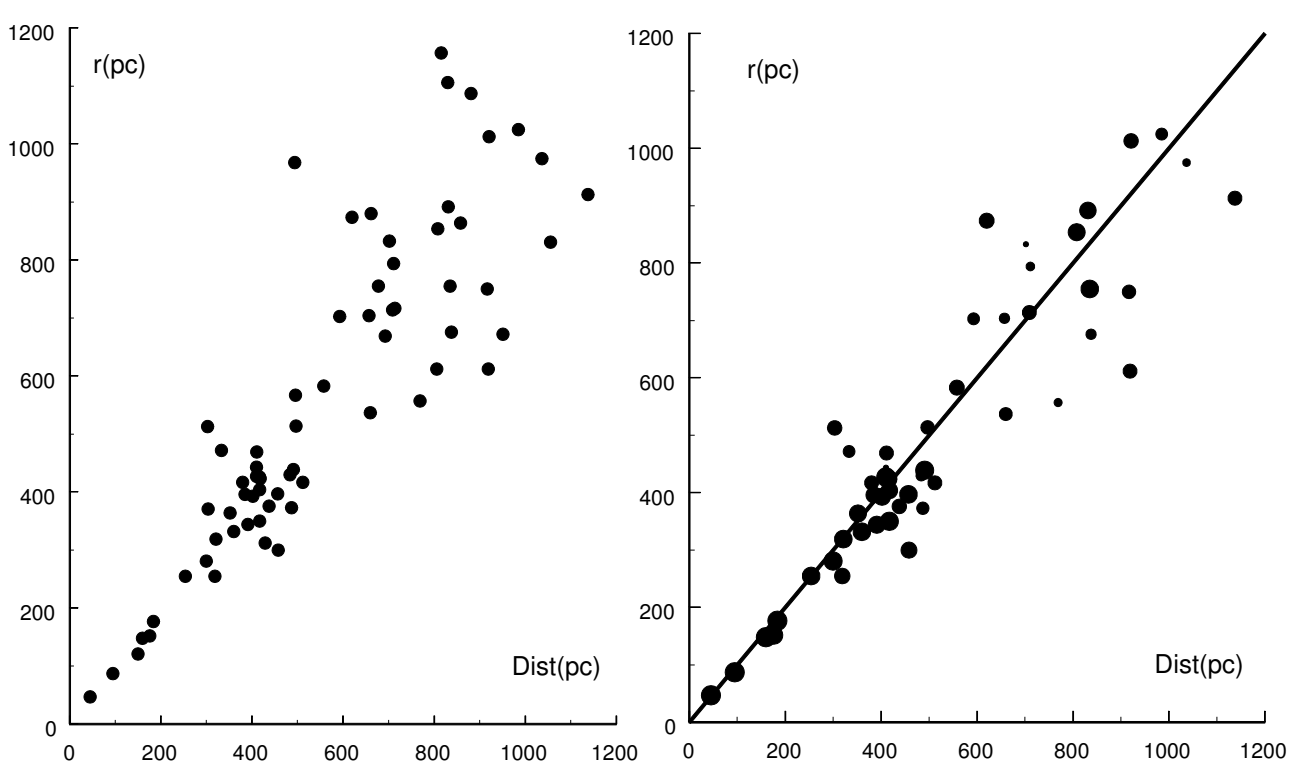

Fig. 4 A comparison of Hipparcos distances (r) with the distances from the "Homogeneous Catalogue" (Dist) for all 68 clusters (left panel) and for doubtful clusters rejected from the sample (right panel). Symbol sizes are proportional to the quality of distance determination from Hipparcos data.

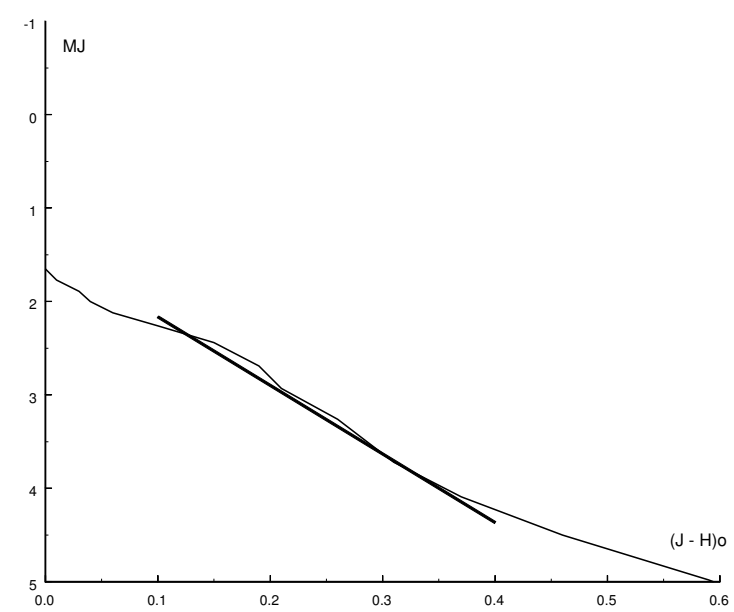

Fig. 5 The agreement of the ZAMS (the $\log t=6.6$ isochrone of Bertelli et al. 1994) and the linear fit for solar metallicity.

lax based $M_{J}$ absolute magnitude errors greater than $0 .{ }^{\mathrm{m}} 3$. Our final sample consists of 727 stars. We applied to this sample a repeated lest-squares fit rejecting the most deviant stars at each step. This allowed us to get rid of stars that have significantly evolved away from the ZAMS. When applied to the 324 stars that remain after the elimination of objects that have evolved from the ZAMS, this procedure yields

$M_{J}=1.43+7.34(J-H)+0.08[\mathrm{Fe} / \mathrm{H}]$,

with the rms errors of the coefficients equal to $0.03,0.10$, and 0.03 , respectively. Figure 5 shows this line compared with ZAMS. The metallicity dependence of the ZAMS appears to be statistically significant but is barely noticeable for the bulk of open clusters.

\section{Discussion}

We show the open cluster distance scale of our "Homogeneous Catalogue" to be well established for both short or long heliocentric distances. The divergent behavior of distance estimates based on near IR photometry is probably caused by factors unrelated to the distance scale. It is most likely due to the asymmetric distribution of faint field stars with respect to the cluster main sequence. This effect is less pronounced in the case of optical photometry because of larger absorption (which is four times stronger in the $V$ band compared to the $J$ band), and it does not affect so strongly the main-sequence fitting procedure in optics. Hence efficient membership assessment units should be incorporated into the new computer program for estimating the cluster parameters from near-IR data.

Acknowledgements. This work is supported in part by the Russian federal task program "Research and operations on priority directions of development of the science and technology complex of Russia for 2007-2013” (contract 14.518.11.7064).

\section{References}

Bertelli, G., Bressan, A., Chiosi, C., Fagotto, F., \& Nasi, E. 1994, A\&AS, 106, 275

Loktin, A. V., \& Matkin, N. V. 1990, SvA, 34, 571

Loktin, A. V., Gerasimenko, T. P., \& Malisheva, L. K. 2001, Astronomical and Astrophysical Transactions, 20, 607

Skrutskie, M. F., Cutri, R. M., Stiening, R., et al. 2006, AJ, 131, 1163

Soubiran, C., Le Campion, J.-F., Cayer de Strobel, G., \& Caillo, A. 2010, A\&A, 515,111

Twarog, B. A., Anthony-Twarog, B. J., \& Edgington-Giordano, F. 2009, PASP, 121, 1312

van Leeuwen, F. 2007, A\&A, 474, 653 